\title{
Anterior vaginal wall length and degree of anterior compartment prolapse seen on dynamic MRI
}

\author{
Yvonne Hsu, \\ Department of Obstetrics and Gynecology, L4000 Women's Hospital, 1500 E Medical Center Drive, \\ Ann Arbor, MI 48109-0276, USA e-mail: yvonneh@med.umich.edu \\ Luyun Chen, \\ Department of Biomedical Engineering, L4000 Women's Hospital, 1500 E Medical Center Drive, \\ Ann Arbor, MI, USA \\ Aimee Summers, \\ Department of Obstetrics and Gynecology, L4000 Women's Hospital, 1500 E Medical Center Drive, \\ Ann Arbor, MI 48109-0276, USA \\ James A. Ashton-Miller, and \\ Department of Biomedical Engineering, L4000 Women's Hospital, 1500 E Medical Center Drive, \\ Ann Arbor, MI, USA \\ James O. L. DeLancey \\ Department of Obstetrics and Gynecology, L4000 Women's Hospital, 1500 E Medical Center Drive, \\ Ann Arbor, MI 48109-0276, USA
}

\section{Abstract}

The objective of the study was to determine the relationship between midsagittal vaginal wall geometric parameters and the degree of anterior vaginal prolapse. We have previously presented data indicating that about half of anterior wall descent can be explained by the degree of apical descent present (Summers et al., Am J Obstet Gynecol, 194:1438-1443, 2006). This led us to examine whether other midsagittal vaginal geometric parameters are associated with anterior wall descent. Magnetic resonance (MR) scans of 145 women from the prior study were suitable for analysis after eight were excluded because of inadequate visibility of the anterior vaginal wall. Subjects had been selected from a study of pelvic organ prolapse that included women with and without prolapse. All patients underwent supine dynamic MR scans in the midsagittal plane. Anterior vaginal wall length, location of distal vaginal wall point, and the area under the midsagittal profile of the anterior vaginal wall were measured during maximal Valsalva. A linear regression model was used to examine how much of the variance in cystocele size could be explained by these vaginal parameters. When both apical descent and vaginal length were considered in the linear regression model, $77 \%\left(R^{2}=0.77\right.$, $p<0.001$ ) of the variation in anterior wall descent was explained. Distal vaginal point and a measure anterior wall shape, the area under the profile of the anterior vaginal wall, added little to the model. Increasing vaginal length was positively correlated with greater degrees of anterior vaginal prolapse during maximal Valsalva $\left(R^{2}=0.30, p<0.01\right)$ determining $30 \%$ of the variation in anterior wall decent. Greater degrees of anterior vaginal prolapse are associated with a longer vaginal wall. Linear regression modeling suggests that $77 \%$ of anterior wall descent can be explained by apical descent and midsagittal anterior vaginal wall length. 


\section{Keywords}

Pelvic organ prolapse; Anterior prolapse; Cystocele; Apical descent; Vaginal length; Dynamic MRI

\section{Introduction}

The anterior vaginal prolapse is the most common form of pelvic organ prolapse [2] as well as the site with the highest rates of persistent and recurrent support defects [3]. Blinded followup in a randomized prospective study of surgical techniques to correct anterior vaginal prolapse at an institution known for excellence in urogynecologic surgery found that only $30-45 \%$ of patients had satisfactory or optimal results at 24 months [4]. The high rate of suboptimal outcomes and the inability to predict which patients will fail surgery suggest a lack of knowledge regarding the disease mechanism of anterior wall prolapse.

Dynamic magnetic resonance imaging (MRI) in the midsagittal plane has given us the ability to study the deformation of the anterior vaginal wall during Valsalva. Previous analysis using this approach found that about half $\left(R^{2}=0.53\right)$ of anterior wall descent can be explained by the degree of apical descent present [1]. The fact that apical descent only explains half of anterior wall descent raises the question of what other changes are involved. This led us to investigate the relationship of other midsagittal geometric factors, such as anterior vaginal wall length, displacement of the distal vagina, and anterior vaginal wall shape, to the degree of anterior wall descent. We hypothesized that these three factors would also predict a significant proportion of the variance in anterior wall descent.

\section{Materials and methods}

A secondary analysis was performed on the sample described in our original report [1]. In brief, we recruited women representing varying degrees of pelvic organ support as part of an ongoing study at the University of Michigan in Ann Arbor, MI. The original study is a casecontrol study where controls with vaginal points at least $1 \mathrm{~cm}$ above the hymen were matched to cases with prolapse at least $1 \mathrm{~cm}$ beyond the hymen [5]. Pelvic support was determined using the pelvic organ prolapse quantification system on clinical exam. Women were selected from this pool to include women in whom the uterus was in situ, the cervix, bladder, and urethra were visible in the dynamic sagittal images, and a correct Valsalva maneuver was performed as judged by the movement of the abdominal wall and intestinal contents in a caudal direction. Women were recruited from the Urogynecology clinic at the University of Michigan and from advertisements. Women were excluded if they had a prior operation for pelvic organ prolapse or urinary incontinence. This resulted in a sample of 153 women for the original analysis. The relationship of the apex, as measured by the cervical os location, and the anterior wall descent, as measured by the most dependent bladder point, was available in these women from the prior study [1]. Magnetic resonance (MR) scans of 145 women from the prior study were suitable for this study with good visualization of the anterior vaginal wall in the dynamic images (mean \pm SD: age $=53.3 \pm 12.5$ years, parity $=2.7 \pm 1.8$, body mass index $=26.4 \pm 4.5 \mathrm{~kg} / \mathrm{m}^{2}$ ). Eight scans from the original study were excluded because the complete vaginal wall could not be adequately seen in sufficient detail for accurate tracing. Although the original study included both cases and controls, our analysis concerned the relationship between cervix location, bladder descent, and vaginal length and did not depend on group status.

MRI was performed on a 1.5-T system (Signa, General Electric, Milwaukee, WI) using a fourchannel torso-phased array coil with the subject in the supine position. Before starting the examination, the patient was instructed in regards to the straining maneuvers to be performed during the examination, starting from minimal to maximal straining. For dynamic imaging, a 
multiphase, single level image of the pelvis in the midsagittal plane was obtained approximately every second for 23-27 s using a T2-weighted single-shot fast spin-echo sequence (time at rest $=1,300 \mathrm{~ms}$, time of excitation $=60 \mathrm{~ms}$, slice thickness $=6 \mathrm{~mm}$, field of view $=32-36 \mathrm{~cm}$, matrix $=256 \times 160$, one excitation and half-Fourier acquisition). The time needed to acquire each of the images was determined by the patients' weight and was approximately a second. A set of 20 successive images were acquired in 23-27 s during rest and a graded Valsalva effort as follows: The operator instructed the subject to hold her breath in inspiration and initiated the scan and after $5 \mathrm{~s}$ of imaging during rest, the operator instructed the subject to strain minimally for $5 \mathrm{~s}$, moderately for $5 \mathrm{~s}$, and maximally for $5 \mathrm{~s}$, then to breath normally and relax for another 5-7 s before ending the acquisition. Usually, three images were acquired at rest during suspended inspiration, 12 during the graded Valsalva effort, and five during post-Valsalva relaxation and normal breathing. These images were placed in a cine-loop using RadPix (Version 3.15, Weadock Software, LLC, Ann Arbor, MI) for display as a movie clip to evaluate the dynamics of prolapse.

The image at maximal Valsalva effort was selected for analysis (Fig. 1). A local coordinate system was created using the inferior pubic point as the origin, the sacrococcygeal inferior pubic point (SCIPP) line as the $x$-axis, and a line perpendicular to the SCIPP line through the inferior pubic point as the $y$-axis (Fig. 1). The location of the external cervical os and the most dependent bladder point was previously determined using this coordinate system [1]. Degree of anterior wall and apical descent were determined by the location of the most caudal bladder point and external cervical os, respectively.

To account for variability in patient size, we normalized all the subjects' pelvises to a SCIPP length of $10.5 \mathrm{~cm}$, which was the mean SCIPP length for our sample. The anterior vaginal wall was traced from the anterior vaginal fornix to the external urinary meatus. A program was created using MatLab (the Mathwork, version 7.01 R14) to measure the length of the vaginal tracings and the SCIPP line. The location of the most distal vaginal point (the ventral end of the vaginal length tracing) and the area under the midsagittal profile of the anterior vaginal wall were also analyzed using MatLab. Finally, the area under the midsagittal anterior wall profile, from its proximal end to its distal end, was measured as the absolute area between the profile and a straight line connecting the two ends of the vaginal wall tracing (Fig. 2). This parameter was a measure of the complexity of the line shape of the vaginal wall tracing. Because these area calculations are a measure of vaginal wall shape rather than a quantification of actual area, non-normalized values were used.

A linear regression model was performed with the dependent variable being prolapse size as measured by the most dependent bladder point and independent variables being the three geometric parameters. We calculated the Pearson correlation coefficient for the regression by comparing the previously obtained most dependent bladder point and the above vaginal wall parameters at maximum Valsalva: $p<0.05$ was considered significant.

\section{Results}

Linear regression (Table 1) showed that $77 \%\left(R^{2}=0.77, p<0.01\right)$ of midsagittal anterior wall descent can be explained by the combination of apical position and vaginal length. The exclusion of the five patients from the original study increased the correlation between cystocele and apical position from $R^{2}=0.53$ to 0.60 . Increasing vaginal length was positively correlated with greater degrees of anterior vaginal prolapse during maximal Valsalva $\left(R^{2}=0.30\right.$, $p<0.01$; Fig. 3$)$. Thus, $30 \%\left(R^{2}=0.30\right)$ of the variation in anterior wall descent was determined by vaginal wall length alone. The area under the vaginal wall profile and the distal urethral location were also investigated for regression modeling but were found not to contribute much to the degree of anterior wall descent. 
As would be expected from the correlation found between apical descent and anterior wall descent, there were subjects who had uterine descent and large cystoceles (Fig. 4a). However, there were also subjects with relatively well-supported apical compartments who had large cystoceles with long anterior vaginal walls (Fig. 4b). Other morphologic variants existed as well where there were examples of patients with cystoceles with normal and short vaginal wall lengths (Fig. 4c, d, respectively).

The relationship between apical descent and vaginal wall length was examined to see if apical descent resulted in longer vaginal length. The correlation was $R^{2}=0.36, p=0.02$.

\section{Discussion}

Linear regression modeling shows that $77 \%$ of anterior wall descent can be explained by both vaginal length and apical descent measured on midsagittal dynamic MR images. A positive correlation was found between anterior wall descent and anterior vaginal wall length with $30 \%$ of variation in anterior wall descent being explained by the length of the vaginal wall. These relationships suggest that the disease mechanism of anterior wall prolapse is likely multifactoral resulting in different morphologic variants of cystocele. Anterior vaginal support has been simplified to a trapezoidal vaginal wall with connections at the top and laterally [6]. Our previous work supports the hypothesis that defects of the apical support are related to cystocele formation [1]. The weak correlation between apical descent and vaginal length and the strong correlation between vaginal length and cystocele size suggest that alterations in the actual vaginal wall itself may be involved in cystocele development.

Vaginal wall characteristics have been previously evaluated. The fraction of smooth muscle in the anterior vaginal wall shows great variability among individuals [7], and its contractility is significantly decreased in women with pelvic organ prolapse [8,9]. Patients with prolapse have also been found to have alterations in collagen ratios and turn over of collagen [10-12]. Findings regarding the collagen content of these tissues have been inconsistent with some studies showing no change [13,14], and others increased content [15]. Comparison of 3D vaginal models of patients with and without prolapse showed similar vaginal anterior-posterior thickness between groups suggesting that the vaginal wall of patients with prolapse is not attenuated [16]. The shear strength of vaginal wall specimens was lower in patients with pelvic floor dysfunction than normal controls [17], and great variability in the tensile and bending strength of samples has also been observed [18].

The vaginal apex has also been examined as the site of failure. One study reported that collagen content was similar between prolapse patients and controls at the vaginal apex but was lower in the parametrial tissue [19]. The resiliency of the apical support structures such as the uterosacral ligaments has also been found to be significantly reduced among women with symptomatic uterovaginal prolapse [20].

Dynamic MRI has been used for the evaluation and staging of pelvic organ prolapse [21-23]. Ozasa et al. [24] also used midsagittal dynamic MRI to asses changes in pelvic support in women with prolapse. They qualitatively compared descent using the relationship of a best-fit levator line and the pubic bone, however, did not perform quantitative measurements. The current study has the advantage of quantifying midsagittal changes that occur during Valsalva. These quantifications deepen our understanding of anterior compartment prolapse mechanisms.

There are several limitations of this study. The dynamic sequence used is limited to a midsagittal view, thereby not allowing the study of changes that may occur in the lateral vaginal wall, which have been observed in anterior compartment prolapse [25]. Therefore, we cannot comment on the presence or effects of paravaginal defects. In addition, these sequences were 
acquired in the supine position, which may limit the descent of the pelvic structures. We minimized this limitation by careful coaching the woman to achieve the maximal prolapse and reviewed all the dynamic video clips to make sure that Valsalva was performed correctly. In the past, researchers have compared supine dynamic MRI with those performed in a sitting position in an open configuration scanner and found supine scans during straining to be comparable in documenting pelvic floor movement [26,27]. An additional limitation is represented by the example in Fig. 4d. This woman has significant anterior wall prolapse and apical descent but yet has a short vaginal length. Although clinically, this woman does not have a shortened wall, she appears to on dynamic MRI because the anterior wall is folded in on itself like an accordion.

The association of increased midline vaginal length in anterior compartment prolapse provides objective data to be considered in the clinical thinking and treatment of cystoceles.

Traditionally, anterior vaginal wall prolapse is attributed to either lateral detachment of the anterior vaginal wall at the pelvic side wall, referred to as a displacement "cystocele," or as a central failure of the vaginal wall itself that results in a distension "cystocele." Whether length changes are due to a paravaginal defect, midline defect, or are simply due to stretching of the vaginal wall once a cystocele becomes present will require further research. The association seen in this study nevertheless raises interesting clinical questions. For example, do traditional side-to-side plications address excess vaginal length associated with anterior compartment prolapse?

\section{Acknowledgments}

We gratefully acknowledge the funding from NIH grant NICHD R01 HD038665 as well as investigator support from 5K12RR017607-04 and the Office for Research on Women's Health and NICHD SCOR on Sex and Gender Factors Affecting Women's Health P50 HD044406. This information was presented at the American Urogynecology Society meeting in October 2006.

\section{References}

1. Summers A, Winkel LA, Hussain HK, DeLancey JOL. The relationship between anterior and apical compartment support. Am J Obstet Gynecol 2006;194:1438-1443. [PubMed: 16579933]

2. Hendrix SL, Clark A, Nygaard I, Aragaki A, Barnabei V, McTiernan A. Pelvic organ prolapse in the Women's Health Initiative: gravity and gravidity. Am J Obstet Gynecol 2002;186(6):1160-1166. [PubMed: 12066091]

3. Shull BL, Bachofen C, Coates KW, Kuehl TJ. A transvaginal approach to repair of apical and other associated sites of pelvic organ prolapse with uterosacral ligaments. Am J Obstet Gynecol 2000;183:1365-1373. [PubMed: 11120498]

4. Weber AM, Walters MD, Piedmonte MR, Ballard LA. Anterior colporrhaphy: a randomized trial of three surgical techniques. Am J Obstet Gynecol 2001;185:1299-1304. [PubMed: 11744900]

5. DeLancey JO, Morgan DM, Fenner DE, Kearney R, Guire K, Miller JM, Hussain H, Umek W, Hsu Y, Ashton-Miller JA. Comparison of levator ani muscle defects and function in women with and without pelvic organ prolapse. Obstet Gynecol 2007;109:295-302. [PubMed: 17267827]

6. DeLancey JOL. Fascial and muscular abnormalities in women with urethral hypermobility and anterior vaginal wall prolapse. Am J Obstet Gynecol 2002;187:93-98. [PubMed: 12114894]

7. Morgan DM, Iyengar J, DeLancey JO. A technique to evaluate the thickness and density of nonvascular smooth muscle in the suburethral fibromuscular layer. Am J Obstet Gynecol 2003;188(5):1183-1185. [PubMed: 12748472]

8. Boreham MK, Wai CY, Miller RT, Schaffer JI, Word RA. Morphometric analysis of smooth muscle in the anterior vaginal wall of women with pelvic organ prolapse. Am J Obstet Gynecol 2002;187(1): 56-63. [PubMed: 12114889] 
9. Boreham MK, Wai CY, Miller RT, Schaffer JI, Word RA. Morphometric properties of the posterior vaginal wall in women with pelvic organ prolapse. Am J Obstet Gynecol 2002;187(6):1501-1508. [PubMed: 12501053]

10. Chen BH, Wen Y, Li H, Polan ML. Collagen metabolism and turnover in women with stress urinary incontinence and pelvic prolapse. Int Urogynecol J Pelvic Floor Dysfunct 2002;13(2):80-87. [PubMed: 12054187]

11. Moalli PA, Talarico LC, Sung VW, Klingensmith WL, Shand SH, Meyn LA, Watkins SC. Impact of menopause on collagen subtypes in the arcus tendineous fasciae pelvis. Am J Obstet Gynecol 2004;190(3):620-627. [PubMed: 15041990]

12. Norton P, Boyd C, Deak S. Collagen synthesis in women with genital prolapse or stress urinary incontinence. Neurourol Urodyn 1992;11:300-301.

13. Liapis A, Bakas P, Pafiti A, Hassiakos D, Frangos-Plemenos M, Creatsas G. Changes in the quantity of collagen type I in women with genuine stress incontinence. Urol Res 2000;28(5):323-326. [PubMed: 11127711]

14. Liapis A, Bakas P, Pafiti A, Frangos-Plemenos M, Arnoyannaki N, Creatsas G. Changes of collagen type III in female patients with genuine stress incontinence and pelvic floor prolapse. Eur J Obstet Gynecol Reprod Biol 2001;97(1):76-79. [PubMed: 11435014]

15. Kokcu A, Yanik F, Cetinkaya M, Alper T, Kandemir B, Malatyalioglu E. Histopathological evaluation of the connective tissue of the vaginal fascia and the uterine ligaments in women with and without pelvic relaxation. Arch Gynecol Obstet 2002;266(2):75-78. [PubMed: 12049299]

16. Hsu Y, Chen L, DeLancey JOL, Ashton-Miller JA. Vaginal thickness, cross-sectional area, and perimeter in women with and those without prolapse. Obstet Gynecol 2005;105(5):1012-1017. [PubMed: 15863538]

17. Kondo A, Narushima M, Yoshikawa Y, Hayashi H. Pelvic fascia strength in women with stress urinary incontinence in comparison with those who are continent. Neurourol Urodyn 1994;13(5):507-513. [PubMed: 7833968]

18. Cosson M, Lambaudie E, Boukerrou M, Lobry P, Crepin G, Ego A. A biomechanical study of the strength of vaginal tissues. Results on 16 post-menopausal patients presenting with genital prolapse. Eur J Obstet Gynecol Reprod Biol 2004;112(2):201-205. [PubMed: 14746960]

19. Takano CC, Girao MJ, Sartori MG, Castro RA, Arruda RM, Simoes MJ, et al. Analysis of collagen in parametrium and vaginal apex of women with and without uterine prolapse. Int Urogynecol J 2002;13(6):342-345.

20. Reay Jones NH, Healy JC, King LJ, Saini S, Shousha S, Allen-Mersh TG. Pelvic connective tissue resilience decreases with vaginal delivery, menopause and uterine prolapse. Br J Surg 2003;90(4): 466-472. [PubMed: 12673750]

21. Etlik O, Arslan H, Odabasi O, Odabasi H, Harman M, Celebi H, et al. The role of the MR-fluoroscopy in the diagnosis and staging of the pelvic organ prolapse. Eur J Radiol 2005;53:136-141. [PubMed: 15607865]

22. Lienemann A, Anthuber C, Baron A, Kohz P, Reiser M. Dynamic MR colpocystorectography assessing pelvic-floor descent. Eur Radiol 1997;7(8):1309-1317. [PubMed: 9377520]

23. Singh K, Reid WM, Berger LA. Assessment and grading of pelvic organ prolapse by use of dynamic magnetic resonance imaging. Am J Obstet Gynecol 2001;185(1):71-77. [PubMed: 11483907]

24. Ozasa H, Mori T, Togashi K. Study of uterine prolapse by magnetic resonance imaging: Topographical changes involving the levator ani muscle and the vagina. Gynecol Obstet Invest 1992;34:43-48. [PubMed: 1526530]

25. Richardson AC, Edmonds PB, Williams NL. Treatment of stress urinary incontinence due to paravaginal fascial defect. Obstet Gynecol 1981;57:357. [PubMed: 7465150]

26. Fielding JR, Griffiths DJ, Versi E, Mulkern RV, Lee MLT, Jolesz FA. MR imaging of pelvic floor continence mechanisms in the supine and sitting positions. Am J Roentgenol 1998;171:1607-1610. [PubMed: 9843296]

27. Bertsschinger KM, Hetzer FH, Roos JE, Treiber K, Marincek B, Hilfiker PR. Dynamic MR imaging of the pelvic floor performed with patient sitting in an open-magnet unit versus with patient supine in a closed-magnet unit. Radiology 2002;223(2):501-508. [PubMed: 11997560] 


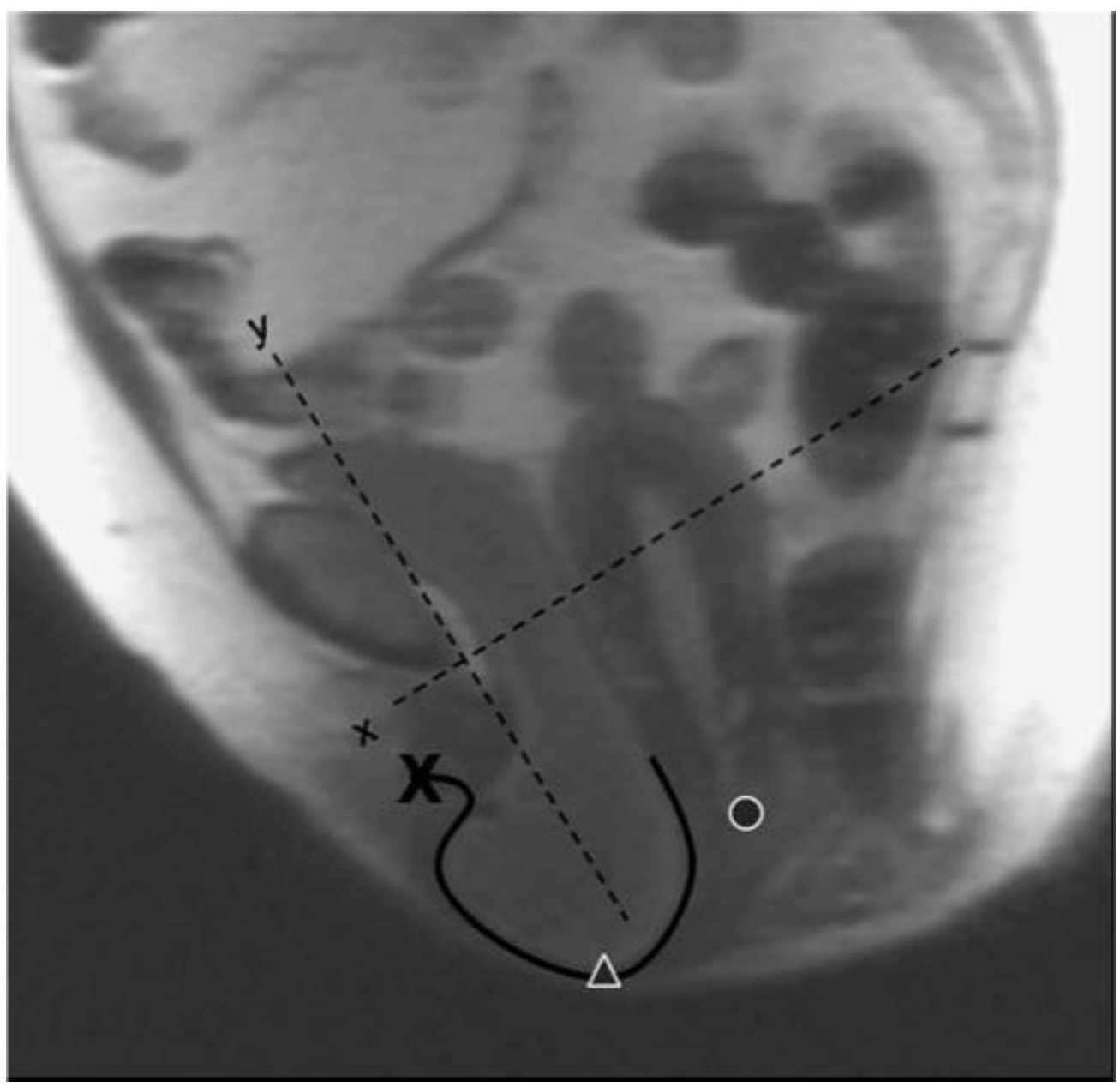

Fig. 1.

MR image at maximal Valsalva showing anterior vaginal wall tracing (solid black line), distal vaginal wall ( $x$ ), bladder location (triangle), cervical os location (circle), and coordinate system (dotted lines) 


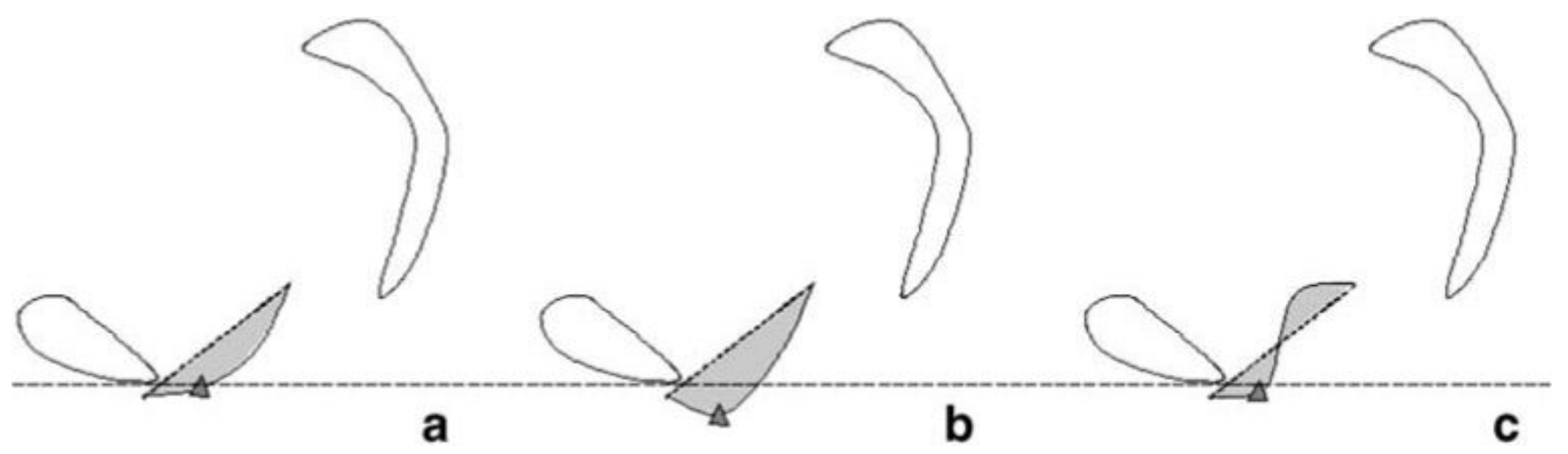

Fig. 2.

Area under the anterior vaginal wall profile. Pubic bone, sacrum, and coccyx are traced. The anterior vaginal wall tracings are shown. A line (small dotted) is used to connect the two ends of the vaginal wall tracing to create an area (shown in gray). Examples aligned using horizontal large dotted reference line. a Minimal descent of most caudal bladder point (triangle). b Greater descent of the bladder point with longer vaginal wall length and larger area. c Similar vaginal wall length to $\mathrm{B}$ but smaller area and lesser descent of bladder point 


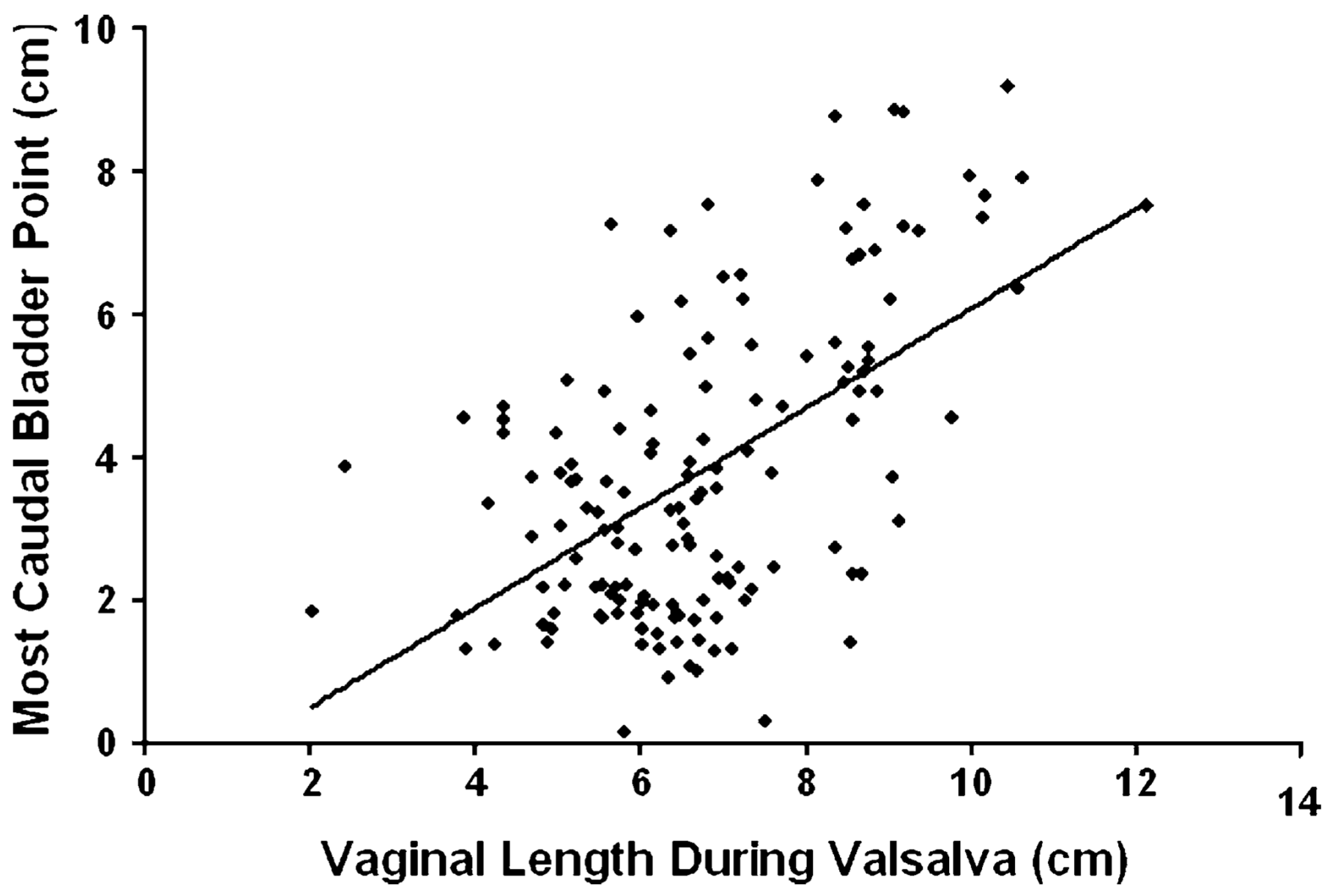

Fig. 3.

Vaginal length during Valsalva and distance of the most caudal bladder point below normal. There is a linear correlation $y=0.69 x-0.85, R^{2}=0.30$ 

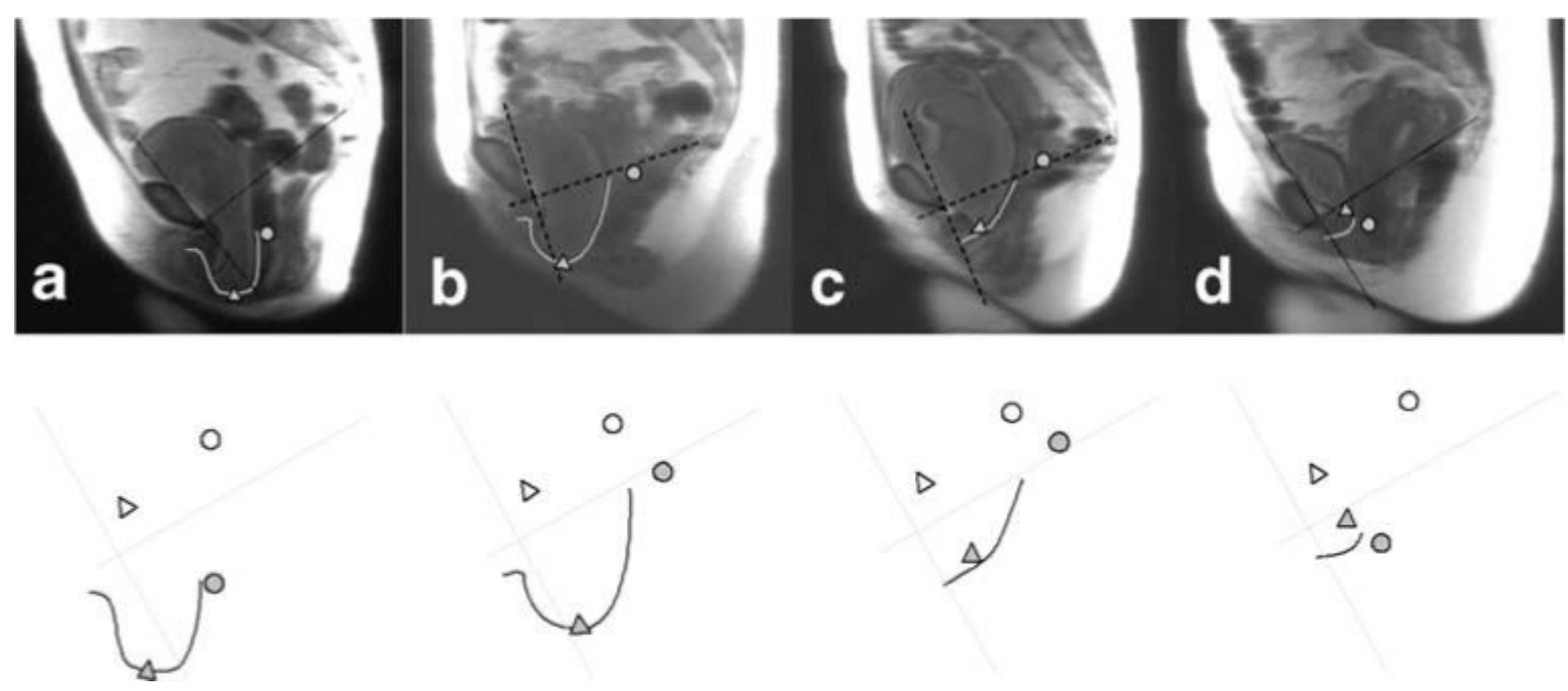

○

Fig. 4.

Subject examples showing relationship between apical support and vaginal length in determining the size of cystoceles. Top row:MR image with maximal Valsalva. Bottom row: Coordinate axis, vaginal tracings, as well as bladder and cervical locations. Images have been standardized for pelvic size and orientation. a Subject who had uterine descent and large cystocele. b Subject with relatively well-supported apical compartments who had large cystocele with a long anterior vaginal wall. c Subject with cystocele and a normal vaginal wall length. d Subject with cystocele with a short vaginal wall length 
Table 1

Linear regression modeling

\begin{tabular}{lllc}
\hline & Cumulative $\boldsymbol{R}^{\mathbf{2}}$ & $\boldsymbol{R}^{\mathbf{2}}$ added & $\boldsymbol{p}$ value \\
\hline Apical & 0.60 & - & $<0.001$ \\
+ Vaginal length & 0.77 & 0.17 & $<0.001$ \\
+Distal point & 0.78 & 0.01 & $<0.001$ \\
+ Area under vaginal profile & 0.81 & 0.03 & $<0.001$ \\
& & & \\
\hline
\end{tabular}

